\title{
DIGITALCOMMONS
}

@WAYNESTATE-

Wayne State University

7-17-2019

\section{On Optimal Stopping and Impulse Control with Constraint}

\author{
J. L. Menaldi \\ Wayne State University, menaldi@wayne.edu \\ M. Robin \\ Fondation Campus Paris-Saclay, maurice.robin@polytechnique.edu
}

Follow this and additional works at: https://digitalcommons.wayne.edu/mathfrp

Part of the Control Theory Commons, and the Other Mathematics Commons

\section{Recommended Citation \\ Menaldi J.L., Robin M. (2019) On Optimal Stopping and Impulse Control with Constraint. In: Yin G., Zhang Q. (eds) Modeling, Stochastic Control, Optimization, and Applications. The IMA Volumes in Mathematics and its Applications, vol 164. Springer, Cham}

This Book Chapter is brought to you for free and open access by the Mathematics at DigitalCommons@WayneState. It has been accepted for inclusion in Mathematics Faculty Research Publications by an authorized administrator of DigitalCommons@WayneState. 


\title{
On optimal stopping and impulse control with constraint October 31, 2018
}

\author{
J.L. Menaldi and M. Robin
}

\begin{abstract}
The optimal stopping and impulse control problems for a Markov-Feller process are considered when the controls are allowed only when a signal arrives. This is referred to as control problems with constraint. Previous results of the authors $[28,29,30]$ are extended to the situation where the state space of the Markov process is locally compact.
\end{abstract}

Keywords: Markov-Feller processes, information constraints, impulse control, control by interventions, ergodic control.

AMS Subject Classification: Primary 49J40 and Secondary 60J60, $60 \mathrm{~J} 75$.

\section{Introduction}

A considerable literature has been devoted to optimal stopping and impulse control of Markov processes (e.g., see the references in Bensoussan and Lions [3, 4], Bensoussan [2], Davis [10]). A relatively small part of this literature concerns problems where constraints are imposed on the admissible stopping times. In the present paper, we address optimal stopping and impulse control problems of a Markov process $x_{t}$ when the stopping times must satisfy a constraint, namely, the control is allowed to take place only at the jump times of a given process $y_{t}$, these times representing the arrival of a signal.

For instance, the system evolves according to a diffusion process $x_{t}$ and the signal $y_{t}$ is a Poisson process as in Dupuis and Wang [11], where an optimal stopping

J.L. Menaldi

Wayne State University, Department of Mathematics, Detroit, MI 48202, USA, e-mail: menal di @ wayne.edu

M. Robin

Fondation Campus Paris-Saclay, 91190 Saint-Aubin, France, e-mail: maurice.robine polytechnique.edu 
problem is studied. In this example, thanks to the memoryless property of the exponential distribution, the $y_{t}$ process does not appear as such. It is interesting to notice that, in the usual (unconstrained) case, the dynamic programming leads to the variational inequality $\max \{-A u+\alpha u, u-\psi\}=0$, where $A$ is the infinitesimal generator of $x_{t}$ and $\psi$ is the stopping cost (with running cost $f=0$ ). However, in the constrained case, this becomes the equation $-A u+\alpha u+\lambda[u-\psi]^{+}=0$, where $\lambda$ is the intensity of the Poisson process (which is assumed independent from $x_{t}$ ). As soon as the intervals between the jumps of $y_{t}$ are not exponentially distributed, the control problem must be formulated with the couple $\left(x_{t}, y_{t}\right)$ and the generator of this two-component process intervenes in the HJB equation.

Such problems has been studied in $[28,29,30]$, when the process $x_{t}$ takes values in a metric compact space $E$ and $y_{t}=t-\tau_{n}$, where $\left\{\tau_{n}\right\}$ is an increasing sequence of instants such that $T_{n}=\tau_{n}-\tau_{n-1}$, for $n \geq 1$ are, conditionally to $x_{t}$, IID random variables. Using an auxiliary discrete time problem in a systematic way, some results have been obtained for optimal stopping and impulse control (with discounted and ergodic cots).

The main aim of the present work is to extend the previous results to the case of a locally compact Polish space, considering the three categories of problems: optimal stopping, impulse control with discounted cost as well as ergodic cost. We also mention further extensions and how some generalizations of the present model is related to hybrid models.

Without pretending to be comprehensive, let us mention (a) that references related to optimal stopping with constraint include also Lempa [23], Liang [25] who studied particular cases of the model considered here and (b) that other class of (analogue) constraint have been considered, e.g., in Egloff and Leippold [12]. Moreover, for impulse control with constraint, we found only a few references, Brémaud [7, 8], Liang and Wei [26], and Wang [39]. A different kind of constraint is considered in Costa et al. [9], where the constraints are written as infinite horizon expected discounted costs.

The paper is organized as follows. In section 2, we introduce notations, definitions and preliminary properties of the uncontrolled process, which is the two components process $\left(x_{t}, y_{t}\right)$. Section 3 presents the definition of the optimal stopping problem and its solution. Section 4 describes the process controlled by impulses and the assumptions, which are used for both discounted cost and ergodic cost. In section 5, the impulse control problem with discounted cost is solved via the HJB equation. In section 6, we present the ergodic cost problem and its solution. Some extension are mentioned in section 7 and in section 8 we discuss the links with hybrid models.

\section{The Uncontrolled Process}

Let us begin with some notations, definitions, comments, and preliminary properties. 


\section{Basic Notations:}

- $\mathbb{R}^{+}=[0, \infty[, E$ a locally compact, separable and complete metric space (in short, a locally compact Polish space), and also $\mathbb{N}_{0}=\{0,1, \ldots\}$ (i.e., natural numbers and 0), $\overline{\mathbb{N}}_{0}=\mathbb{N}_{0} \cup\{\infty\}, \overline{\mathbb{R}}^{+}=[0, \infty]$;

- $\mathscr{B}(Z)$ the Borel $\sigma$-algebra of sets in $Z, B(Z)$ the space of real-valued Borel and bounded functions on $Z, C_{b}(Z)$ the space of real-valued continuous and bounded functions on $Z, C_{0}(Z)$ real-valued continuous functions vanishing at infinity on $Z$, i.e., a real-valued continuous function $v$ belongs to $C_{0}(Z)$ if and only if for every $\varepsilon>0$ there exists a compact set $K$ of $Z$ such that $|v(z)|<\varepsilon$ for every $z$ in $Z \backslash K^{1}$, and also, if necessary, $B^{+}(Z), C_{b}^{+}(Z), C_{0}^{+}(Z)$ for non-negative functions; usually either $Z=E$ or $Z=E \times \mathbb{R}^{+}$;

- the canonical space $D\left(\mathbb{R}^{+}, Z\right)$ of cad-lag functions, with its canonical process $z_{t}(\omega)=\omega(t)$ for any $\omega \in D\left(\mathbb{R}^{+}, Z\right)$, and its canonical filtration $\mathbb{F}^{0}=\left\{\mathscr{F}_{t}^{0}: t \geq\right.$ $0\}, \mathscr{F}_{t}^{0}=\sigma\left(z_{s}: 0 \leq s \leq t\right)$.

Assumption 2.1 Let $\left(\Omega, \mathbb{F}, x_{t}, y_{t}, P_{x y}\right)$ be a (realization of a) strong and normal homogeneous Markov process, on $\Omega=D\left(\mathbb{R}^{+}, E \times \mathbb{R}^{+}\right)$with its canonical filtration universally completed $\mathbb{F}=\left\{\mathscr{F}_{t}: t \geq 0\right\}$ with $\mathscr{F}_{\infty}=\mathscr{F}$, where $\left(x_{t}, y_{t}\right)$ is the canonical process having values in $E \times \mathbb{R}^{+}$, and $\mathbb{E}_{x y}$ (or $\mathbb{E}_{x, y}$ when a confusion may arrive) denotes the expectation relative to $P_{x y}$.

a) It is also assumed that $x_{t}$ is a Markov process by itself (referred as the reduced state), with a $C_{0}$-semigroup $\Phi_{x}(t)$ (i.e., $\Phi_{x}(t) C_{0}(E) \subset C_{0}(E), \forall t \geq 0$ ), and infinitesimal generator $A_{x}$ with domain $\mathscr{D}\left(A_{x}\right) \subset C_{0}(E)$.

b) The process $y_{t}$ (referred to as the signal process) has jumps to zero at times $\tau_{1}, \ldots, \tau_{n} \rightarrow \infty$ and $y_{t}=t-\tau_{n}$ for $\tau_{n} \leq t<\tau_{n+1}$ (i.e., $\tau_{1}$ is the time of the first jump-to zero- of $y_{t}$, each jump is 'the signal' and $y_{t}$ is exactly the 'time elapsed since the last jump or signal'), and if $y_{0}=0$ and $\tau_{0}=0$ then it is assumed that conditionally to $x_{t}$, the intervals between jumps $T_{n}=\tau_{n}-\tau_{n-1}$ are independent, identically distributed random variables with a non-negative continuous and bounded intensity function $\lambda(x, y)$, which is such that there exists a constant $K>0$ satisfying $\mathbb{E}_{x 0}\left\{\tau_{1}\right\} \leq K$, for any $x$ in $E$.

Remark 2.1. Actually, we begin with a realization of the reduced state process $x_{t}$ on the canonical space $D\left(\mathbb{R}^{+}, E\right)$ and the signal process $y_{t}$ is constructed based on the given intensity $\lambda(x, y)$, and this procedure yields a $C_{0}\left(E \times \mathbb{R}^{+}\right)$-semigroup denoted by $\Phi_{x y}(t)$. Thus, in view of Palczewski and Stettner [34], all this implies that both semigroups $\Phi_{x}(t)$ and $\Phi_{x y}(t)$ have the Feller property, i.e., $\Phi_{x y}(t) C_{b}(E) \subset C_{b}(E)$ and $\Phi_{x y}(t) C_{b}\left(E \times \mathbb{R}^{+}\right) \subset C_{b}\left(E \times \mathbb{R}^{+}\right)$, and since only a strong and normal Markov process is assumed, the semigroup $\Phi_{x y}(t)$ is (initially) acting on $B\left(E \times \mathbb{R}^{+}\right)$and so, weak (or stochastic) continuity is deduced from the assumption of a cad-lag realization, which means that

$$
(x, y, t) \mapsto \mathbb{E}_{x y}\left\{h\left(x_{t}, y_{t}\right)\right\} \quad \text { is a continuous function, }
$$

\footnotetext{
${ }^{1}$ Typically $E=\mathbb{R}^{d}$ and this means that $v(z) \rightarrow 0$ as $|z| \rightarrow \infty$.
} 
for any $h$ in $C_{b}\left(E \times \mathbb{R}^{+}\right)$. In [28, 29,30] a probabilistic construction of the signal process $y_{t}$ was described, but there are other ways to constructing $\Phi_{x y}(t)$. For instances, begin with the process $\left(x_{t}, \tilde{y}_{t}\right)$ with $\tilde{y}_{t}=y+t$ having infinitesimal generator $A^{0}=A_{x}+\partial_{y}$ and a $C_{0}\left(E \times \mathbb{R}^{+}\right)$-semigroup. Then, add the perturbation $B h(x, y)=\lambda(x, y)[h(x, 0)-h(x, y)]$, which is a bounded operator generating a $C_{0}\left(E \times \mathbb{R}^{+}\right)$-semigroup, with domain $\mathscr{D}(B)=C_{0}\left(E \times \mathbb{R}^{+}\right)$. Hence $A_{x y}=A^{0}+B$ generates a $C_{0}\left(E \times \mathbb{R}^{+}\right)$-semigroup, with $\mathscr{D}\left(A_{x y}\right)=\mathscr{D}\left(A^{0}\right)$, e.g., see Ethier and Kurtz [13, Section 1.7, pp. 37-40, Thm 7.1]. Therefore $A_{x y}$ will also denote the weak infinitesimal generator in $C_{b}\left(E \times \mathbb{R}^{+}\right)$, in several places of the following sections.

Remark 2.2. Note that Assumption 2.1 (b) on the signal process $y_{t}$ means, in particular, that

$$
P_{x 0}\left\{T_{n} \in(t, t+d t) \mid x_{s}, 0 \leq s \leq t\right\}=\lambda\left(x_{t}, t\right) \exp \left(-\int_{0}^{t} \lambda\left(x_{s}, s\right) \mathrm{d} s\right),
$$

and then it is deduced that $\Phi_{x y}(t)$ has an infinitesimal generator $A_{x y}=A_{x}+A_{y}$ with

$$
A_{y} \varphi(x, y)=\partial_{y} \varphi(x, y)+\lambda(x, y)[\varphi(x, 0)-\varphi(x, y)],
$$

and recall that $\partial_{y}$ denotes the derivative with respect to $y$, and that $\lambda \geq 0$ and $\lambda \in$ $C_{b}\left(E \times \mathbb{R}^{+}\right)$. Moreover, using the law of $T_{1}$ as in (2) and the Feller property of $\left(x_{t}, y_{t}\right)$, it is also deduced that

$$
(x, y) \mapsto \mathbb{E}_{x y}\left\{\mathrm{e}^{-\alpha \tau_{1}} g\left(x_{\tau_{1}}\right)\right\} \text { belongs to } C_{b}\left(E \times \mathbb{R}^{+}\right),
$$

for any $g$ in $C_{b}(E)$ and any $\alpha \geq 0$. Note that if $y_{0}=y$ then $\tau_{1}$ is random variable independent of $T_{1}, T_{2}, \ldots$ with distribution $P_{x 0}\left\{T_{1} \in \cdot \mid y_{0}=y\right\}$. Furthermore, in turn, by applying Dynkin's formula to $A_{x y} \varphi(x, y)+\alpha \varphi(x, y)=f(x, y)$, it follows that

$$
(x, y) \mapsto \mathbb{E}_{x y}\left\{\int_{0}^{\tau_{1}} \mathrm{e}^{-\alpha t} f\left(x_{t}, y_{t}\right) \mathrm{d} t\right\} \text { is in } C_{b}\left(E \times \mathbb{R}^{+}\right),
$$

for any $f$ in $C_{b}\left(E \times \mathbb{R}^{+}\right)$and any $\alpha>0$.

Remark 2.3. Note that because $\lambda(x, y)$ is bounded (it suffices for $y$ near 0 ), there exists a constant $a$ such that $P_{x 0}\left\{\tau_{1} \geq a>0\right\} \geq a>0$, for any $x$ in $E$. Moreover, from Assumption 2.1 (b) on the signal process $y_{t}$ we have

$$
\mathbb{E}_{x 0}\left\{\tau_{1}\right\}=\mathbb{E}_{x 0}\left\{\int_{0}^{\infty} t \lambda\left(x_{t}, t\right) \exp \left(-\int_{0}^{t} \lambda\left(x_{s}, s\right) \mathrm{d} s\right) \mathrm{d} t\right\},
$$

so if $\lambda(x, y) \leq k_{1}<\infty$, for every $y \geq 0$, and $x \in E$, then $\mathbb{E}_{x 0}\left\{\tau_{1}\right\} \geq a_{1}=1 / k_{1}$.. Also, the condition $\mathbb{E}_{x 0}\left\{\tau_{1}\right\} \leq a_{2}$ is satisfied if, for instance $\lambda(x, y) \geq k_{0}>0$ for $y \geq y_{0}, x \in E$, then $a_{2}=y_{0}+1 / k_{0}$. Moreover, since $\lambda(x, y)$ is a continuous function in $E \times \mathbb{R}^{+}$, the continuity of $E_{x y}\left\{\tau_{1}\right\}$ follows. 
Definition 2.1 (with comments). If the evolution $\dot{e}=-\alpha t$ in $[0,1]$ is added to the homogeneous Markov process $\left\{\left(x_{t}, y_{t}\right): t \geq 0\right\}$ then the expression

$$
\left\{\left(X_{n}, e_{n}\right)=\left(x_{\tau_{n}}, \mathrm{e}^{-\alpha \tau_{n}}\right), n=0,1, \ldots\right\},
$$

with $e_{0}=1, \tau_{0}=0$ and $X_{0}=x$, becomes a homogeneous Markov chain in $\left.] 0,1\right] \times E$ with respect to the filtration $\mathbb{G}=\left\{\mathscr{G}_{n}: n=0,1, \ldots\right\}$ obtained from $\mathbb{F}$, namely, $\mathscr{G}_{n}=$ $\mathscr{F}_{\tau_{n}}$. Note that $\left\{x_{\tau_{n}}: n \geq 0\right\}$ is also a Markov chain with respect to $\mathscr{G}_{n}$. In this context, if

$$
\tau=\inf \left\{t>0: y_{t}=0\right\}
$$

is considered as a functional on $\Omega$, then the sequence of signals (i.e., the instants of jumps for $y_{t}$ ) is defined by recurrence

$$
\tau_{k+1}=\inf \left\{t>\tau_{k}: y_{t}=0\right\}, \quad \forall k=1,2, \ldots,
$$

with $\tau_{1}=\tau$, and by convenience, set $\tau_{0}=0$. Let us also mention that Remark 2.3 yields: there exists a constant $a_{1}$ such that

$$
P_{x 0}\left\{\tau \geq a_{1}>0\right\} \geq a_{1}>0, \quad \forall x \in E,
$$

and by Assumption 2.1, there exists another constant $a_{2}>0$ such that

$$
\mathbb{E}_{x 0}\{\tau\} \leq a_{2}, \quad \forall x \in E .
$$

It is also valid,

$$
0<a_{1} \leq \tau(x):=\mathbb{E}_{x 0}\{\tau\} \leq a_{2}, \quad \forall x \in E,
$$

for some real numbers $a_{1}, a_{2}$. An $\mathbb{F}$-stopping time $\theta>0$ satisfying $y_{\theta}=0$ when $\theta<\infty$ is called an admissible stopping time, in other words, if and only if there exists a discrete (i.e., $\overline{\mathbb{N}}_{0}$-valued) $\mathbb{G}$-stopping time $\eta$ such that $\theta=\tau_{\eta}$ with the convention that $\tau_{\infty}=\infty$. Moreover, if the condition $\theta>0$ (or equivalently $\eta \geq 1$ ) is dropped then $\theta$ is called a zero-admissible stopping time.

\section{Optimal Stopping with Constraint}

This section is an extension of [28] to a locally compact space $E$. 


\subsection{Setting-up}

The usual optimal stopping problems as presented above is well known, but our interest here is to restrict the stopping action (of the controller) to certain instants when a signal arrives. As discussed in the previous section, the state of the dynamic system is a homogeneous Markov process $\left\{\left(x_{t}, y_{t}\right): t \in \mathbb{R}^{+}\right\}$with values in the locally compact Polish space $E \times \mathbb{R}^{+}$, satisfying the Feller conditions (1). Suppose that

$$
f \in C_{b}\left(E \times \mathbb{R}^{+}\right), \quad \psi \in C_{b}(E), \quad \alpha>0,
$$

where $f(x, y)$ is the running cost, $\psi(x)$ is the terminal cost, and $\alpha$ is the discount factor.

Thus, for any stopping time $\theta$

$$
J_{x y}(\theta, \psi)=\mathbb{E}_{x y}\left\{\int_{0}^{\theta} \mathrm{e}^{-\alpha t} f\left(x_{t}, y_{t}\right) \mathrm{d} t+\mathrm{e}^{-\alpha \theta} \psi\left(x_{\theta}\right)\right\},
$$

is the cost function with the optimal cost

$$
u(x, y)=\inf \left\{J_{x y}(\theta, \psi): \theta>0, y_{\theta}=0\right\}
$$

i.e., $\theta$ is any admissible stopping time, as defined in Section 2. Also, it is defined an auxiliary problem with optimal cost

$$
u_{0}(x, y)=\inf \left\{J_{x y}(\theta, \psi): y_{\theta}=0\right\}
$$

which provides a homogeneous Markovian model. Since $u(x, y)=u_{0}(x, y)$ for any $x \in E$ and $y>0$, it may be convenient to write $u_{0}(x)=u_{0}(x, 0)$ as long as no confusion arrives.

Remark 3.1. Both costs $u(x, y)$ and $u_{0}(x, y)$ represent the optimization over all stopping times that occur when the signal arrives, the difference is that for $y=0$ and $t=0$ (i.e., when the first signal arrives at the beginning), the control action is allowed for the optimal cost $u_{0}(x, 0)$, but it is not allowed for the optimal cost $u(x, 0)$, i.e., one may say that for $u(x, 0)$ the 'controller' is (so to speak) always 'late' (at the beginning and arriving simultaneously with the signal) and control is not possible. One may consider even an alternative situation, where with a certain probability (independently of $\left(x_{t}, y_{t}\right)$, for instance) the control is allowed, and therefore, the optimal cost (in the simplest case) would be a convex combination of $u(x, 0)$ and $u_{0}(x, 0)$. Clearly, all this comment will apply later, for the impulse control problem.

The Dynamic Programming Principle shows (heuristically) that

$$
u(x, y)=\mathbb{E}_{x y}\left\{\int_{0}^{\tau} \mathrm{e}^{-\alpha t} f\left(x_{t}, y_{t}\right) \mathrm{d} t+\mathrm{e}^{-\alpha \tau} \min \{\psi, u\}\left(x_{\tau}, y_{\tau}\right)\right\}
$$

with $\tau=\inf \left\{t>0: y_{t}=0\right\}$ being the first jump of $y_{t}$, and 


$$
\begin{aligned}
& u_{0}(x, y)=\mathbb{E}_{x y}\left\{\int_{0}^{\tau} \mathrm{e}^{-\alpha t} f\left(x_{t}, y_{t}\right) \mathrm{d} t+\mathrm{e}^{-\alpha \tau} u_{0}\left(x_{\tau}, y_{\tau}\right)\right\}, \quad y>0, \\
& u_{0}(x, 0)=\min \left\{\mathbb{E}_{x 0}\left\{\int_{0}^{\tau} \mathrm{e}^{-\alpha t} f\left(x_{t}, y_{t}\right) \mathrm{d} t+\mathrm{e}^{-\alpha \tau} u_{0}\left(x_{\tau}, y_{\tau}\right)\right\}, \psi(x)\right\},
\end{aligned}
$$

are the corresponding Hamilton-Jacobi-Bellman (HJB) equations, which are referred to as variational inequalities (VI) in a weak form. Also, both problems are (logically) related by the condition

$$
u(x, y)=\mathbb{E}_{x y}\left\{\int_{0}^{\tau} \mathrm{e}^{-\alpha t} f\left(x_{t}, y_{t}\right) \mathrm{d} t+\mathrm{e}^{-\alpha \tau} u_{0}\left(x_{\tau}, y_{\tau}\right)\right\} .
$$

Thus, $y_{\tau}=0$ implies

$$
\begin{aligned}
& u_{0}(x)=\min \left\{\psi(x), \mathbb{E}_{x 0}\left\{\int_{0}^{\tau} \mathrm{e}^{-\alpha t} f\left(x_{t}, y_{t}\right) \mathrm{d} t+\mathrm{e}^{-\alpha \tau} u_{0}\left(x_{\tau}\right)\right\}\right\}, \\
& u(x, y)=\mathbb{E}_{x y}\left\{\int_{0}^{\tau} \mathrm{e}^{-\alpha t} f\left(x_{t}, y_{t}\right) \mathrm{d} t+\mathrm{e}^{-\alpha \tau} \min \{\psi, u\}\left(x_{\tau}, 0\right)\right\}, \\
& u(x, y)=\mathbb{E}_{x y}\left\{\int_{0}^{\tau} \mathrm{e}^{-\alpha t} f\left(x_{t}, y_{t}\right) \mathrm{d} t+\mathrm{e}^{-\alpha \tau} u_{0}\left(x_{\tau}\right)\right\},
\end{aligned}
$$

i.e., if $u_{0}(x)$ is known then the above equalities yield $u(x, y)$ and $u_{0}(x, y)$.

\subsection{Solving the VI}

By means of (6), the continuous-time cost $J_{x 0}(\theta, \psi)$ with $f=0$ and a stopping time $\theta=\tau_{\eta}$ can be written as

$$
\begin{aligned}
J_{x 0}(\theta, \psi)=\mathbb{E}_{x 0}\left\{\mathrm{e}^{-\alpha \theta} \psi\left(x_{\theta}\right)\right\} & = \\
= & \mathbb{E}\left\{e_{\eta} \psi\left(X_{\eta}\right) \mid e_{0}=1, X_{0}=x\right\}:=K_{1 x}(\eta, \psi),
\end{aligned}
$$

for any discrete stopping time $\eta$ relative to the Markov chain, i.e., where $\eta$ has values in $\mathbb{N}_{0}=$ and the convention $\tau_{\infty}=\infty$, and the last equality is the definition of the discrete cost $K_{1 x}(\eta, \psi)$. This means that the optimal cost $u_{0}(x)$ is also the optimal cost of a discrete-time stopping time problem relative to the homogeneous Markov chain (certainly, there are several other ways of considering an equivalent problem in discrete-time), i.e, $u_{0}(x)=\inf \left\{K_{1 x}(\eta, \psi): \eta \geq 0\right\}$. This yields

$$
u_{0}(x)=\min \left\{\psi(x), \mathbb{E}_{x 0} \mathrm{e}^{-\alpha \tau} u_{0}\left(x_{\tau}\right)\right\}
$$

as the HJB equation for $u_{0}(x)$, when $f=0$.

Theorem 3.1. Under Assumption 2.1 and (12), the VI (17) and (16) have each a unique solution in $C_{b}\left(E \times \mathbb{R}^{+}\right)$, which are the optimal costs (14) and (15), respectively. Moreover, the first admissible exit time of the continuation region is optimal, 
i.e., the discrete stopping times

$$
\begin{aligned}
& \hat{\theta}=\inf \left\{t>0: u\left(x_{t}, y_{t}\right) \leq \psi\left(x_{t}, y_{t}\right), y_{t}=0\right\}, \\
& \hat{\theta}_{0}=\inf \left\{t \geq 0: u_{0}\left(x_{t}, y_{t}\right)=\psi\left(x_{t}, y_{t}\right), y_{t}=0\right\}
\end{aligned}
$$

are optimal, namely, $u(x, y)=J_{x y}(\hat{\theta}, \psi)$ and $u_{0}(x, y)=J_{x y}\left(\hat{\theta}_{0}, \psi\right)$. Furthermore, the relation (18) holds.

Proof. This result is proved in [28] when $E$ is compact, and it is valid under the assumptions in Section 2 with the same arguments, and therefore, only the main idea and comments are presented.

First, let us mention that the translation

$$
u \longmapsto u-\mathbb{E}_{x y}\left\{\int_{0}^{\infty} \mathrm{e}^{-\alpha t} f\left(x_{t}, y_{t}\right) \mathrm{d} t\right\}
$$

(and similarly with $u_{0}$ ) reduces to a zero running cost, i.e., in all this section we may assume $f=0$ without any lost of generality, only the terminal cost $\psi$ is relevant. Also, Assumption 2.1(b) on the signal and the inequality

$$
\begin{aligned}
\left(1-\mathrm{e}^{-\alpha a}\right) P_{x 0}\{\tau \geq a\}=\left(1-\mathrm{e}^{-\alpha a}\right) P_{x 0}\left\{1-\mathrm{e}^{-\alpha \tau}\right. & \left.\geq 1-\mathrm{e}^{-\alpha a}\right\} \leq \\
& \leq 1-\mathbb{E}_{x 0}\left\{\mathrm{e}^{-\alpha \tau}\right\}, \quad \forall a>0,
\end{aligned}
$$

imply $\mathbb{E}_{x 0}\left\{\mathrm{e}^{-\alpha \tau}\right\} \leq 1-\left(1-\mathrm{e}^{-\alpha a_{1}}\right) a_{0}:=k_{1}<1$. This is used to solve the VI

$$
u_{0}(x)=\min \left\{\mathbb{E}_{x 0}\left\{\int_{0}^{\tau} \mathrm{e}^{-\alpha t} f\left(x_{t}, y_{t}\right) \mathrm{d} t+\mathrm{e}^{-\alpha \tau} u_{0}\left(x_{\tau}\right)\right\}, \psi(x)\right\}
$$

by means of a fixed point for a contraction operator. Then, some martingale arguments are used to establish that $u_{0}(x)$ is indeed the optimal cost of a discrete-time optimal stopping time problem relative to a Markov chain (6), where the first exit time of the continuation region $\left\{x: u_{0}(x)<\psi(x)\right\}$ is optimal. Next, this is connected with the continuous-time problem and the conclusion follows.

If the function $u_{0}(x, y)$ belongs to the domain $\mathscr{D}\left(A_{x y}\right)$ then VI becomes

$$
\begin{aligned}
& \left.A_{x y} u_{0}(x, y)-\alpha u_{0}(x)+f(x, y)=0, \quad \forall(x, y) \in E \times\right] 0, \infty[, \\
& \min \left\{A_{x y} u_{0}(x, y)-\alpha u_{0}(x)+f(x, y), \psi(x)-u_{0}(x, y)\right\}=0, \quad \forall(x, y) \in E \times\{0\},
\end{aligned}
$$

where $A_{x y}$ is the infinitesimal generator. It may be proved that this is indeed the case when $\psi$ also belongs to $\mathscr{D}\left(A_{x y}\right)$, but only continuity is usually not sufficient. However, the optimal cost $u$ given by (14) belongs to $\mathscr{D}\left(A_{x y}\right)$ and the VI (16) is equivalent to

$$
-A_{x y} u(x, y)+\alpha u(x, y)+\lambda(x, y)[u(x, 0)-\psi(x, y)]^{+}=f(x, y),
$$


for any $(x, y)$ in $E \times[0, \infty$, where $\lambda(x, y)$ is the jump-intensity as discussed in the previous section. Also remark $u_{0}=\min \{u, \psi\}$, which makes clear that $u_{0}(x, y)$ may not belong to the domain $\mathscr{D}\left(A_{x y}\right) \subset C_{b}(E \times[0, \infty[)$.

Remark 3.2. The VI/HJB equation (22) is similar to the penalized equation of the unconstrained problem, e.g., see Bensoussan and Lions [3]. Similarly, using the same method as in the penalized problem, if $\lambda$ goes to infinity (uniformly) then the solution $u_{\lambda}$ converges to the solution (which is a function of $x$ only) of the classical variational inequality of the unconstrained problem.

There are some references regarding the stopping time problem with Poisson constraint (e.g., Dupuis and Wang [11], Lempa [23], Liang and Wei [26]), while there are many more about the usual or standard stopping times problem (e.g., the books by Bensoussan and Lions [3], Peskir and Shiryaev [35], among several others books and papers).

\section{Impulse Controlled Process}

This section describes the controlled process and assumptions common to both, the discounted problem and the ergodic problem, as treated in the next two sections.

\subsection{Controlled Process}

For a detailed construction we refer to Bensoussan and Lions [4] (see also Davis [10], Lepeltier and Marchal [24], Robin [36], Stettner [38]).

Let us consider $\Omega^{\infty}=\left[D\left(\mathbb{R}^{+} ; E \times \mathbb{R}^{+}\right)\right]^{\infty}$, and define $\mathscr{F}_{t}^{0}=\mathscr{F}_{t}$ and $\mathscr{F}_{t}^{n+1}=$ $\mathscr{F}_{t}^{n} \otimes \mathscr{F}_{t}$, for $n \geq 0$, where $\mathscr{F}_{t}$ is the universal completion of the canonical filtration as previously.

An arbitrary impulse control $v$ (not necessarily admissible at this stage) is a sequence $\left(\theta_{n}, \xi_{n}\right)_{n \geq 1}$, where $\theta_{n}$ is a stopping time of $\mathscr{F}_{t}^{n-1}, \theta_{n} \geq \theta_{n-1}$, and the impulse $\xi_{n}$ is $\mathscr{F}_{\theta_{n}}^{n-1}$ measurable random variable with values in $E$.

The coordinate in $\Omega^{\infty}$ has the form $\left(x_{t}^{0}, y_{t}^{0}, x_{t}^{1}, y_{t}^{1}, \ldots, x_{t}^{n}, y_{t}^{n}, \ldots\right)$, and for any impulse control $v$ there exists a probability $P_{x y}^{v}$ on $\Omega^{\infty}$ such that the evolution of the controlled process $\left(x_{t}^{v}, y_{t}^{v}\right)$ is given by the coordinates $\left(x_{t}^{n}, y_{t}^{n}\right)$ of $\Omega^{\infty}$ when $\theta_{n} \leq t<\theta_{n+1}, n \geq 0$ (setting $\theta_{0}=0$ ), i.e., $\left(x_{t}^{v}, y_{t}^{v}\right)=\left(x_{t}^{n}, y_{t}^{n}\right)$ for $\theta_{n} \leq t<\theta_{n+1}$. Note that clearly $\left(x_{t}^{v}, y_{t}^{v}\right)$ is defined for any $t \geq 0$, but $\left(x_{t}^{i}, y_{t}^{i}\right)$ is only used for any $t \geq \theta_{i}$, and $\left(x_{\theta_{i}}^{i-1}, y_{\theta_{i}}^{i-1}\right)$ is the state at time $\theta_{i}$ just before the impulse (or jump) to $\left(\xi_{i}, y_{\theta_{i}}^{i-1}\right)=\left(x_{\theta_{i}}^{i}, y_{\theta_{i}}^{i}\right)$, as long as $\theta_{i}<\infty$. For the sake of simplicity, we will not always indicate, in the sequel, the dependency of $\left(x_{t}^{v}, y_{t}^{v}\right)$ with respect to $v$. A Markov impulse control $v$ is identified by a closed subset $S$ of $E \times \mathbb{R}^{+}$and a Borel measurable function $(x, y) \mapsto \xi(x, y)$ from $S$ into $C=E \times \mathbb{R}^{+} \backslash S$, with the following 
meaning: intervene only when the the process $\left(x_{t}, y_{t}\right)$ is leaving the continuation region $C$ and then apply an impulse $\xi(x, y)$, while in the stopping region $S$, moving back the process to the continuation region $C$, i.e., $\theta_{i+1}=\inf \left\{t>\theta_{i}:\left(x_{t}^{i}, y_{t}^{i}\right) \in S\right\}$, with the convention that $\inf \{\emptyset\}=\infty$, and $\xi_{i+1}=\xi\left(x_{\theta_{i+1}}^{i}, y_{\theta_{i+1}}^{i}\right)$, for any $i \geq 0$, as long as $\theta_{i}<\infty$.

Now, the admissible controls are defined as follows, recalling that $\tau_{n}$ are the arrival times of signal

Definition 4.1. (i) As mentioned earlier, a stopping time $\theta$ is called 'admissible' if almost surely there exists $n=\eta(\omega) \geq 1$ such that $\theta(\omega)=\tau_{\eta(\omega)}(\omega)$, or equivalently if $\theta$ satisfies $\theta>0$ and $y_{\theta}=0$ a.s.

(ii) An impulse control $v=\left\{\left(\theta_{i}, \xi_{i}\right), i \geq 1\right\}$ as above is called 'admissible', if each $\theta_{i}$ is admissible (i.e., $\theta_{i}>0$ and $y_{\theta_{i}}=0$ ), and $\xi_{i} \in \Gamma\left(x_{\theta_{i}}^{i-1}\right)$. The set of admissible impulse controls is denoted by $\mathscr{V}$.

(iii) If $\theta_{1}=0$ is allowed, then $v$ is called 'zero-admissible'. The set of zeroadmissible impulse controls is denoted by $\mathscr{V}_{0}$.

(iv) An 'admissible Markov' impulse control corresponds to a stopping region $S=S_{0} \times\{0\}$ with $S_{0} \subset E$, and an impulse function satisfying $\xi(x, 0)=\xi_{0}(x) \in \Gamma(x)$, for any $x \in S_{0}$, and therefore, $\theta_{i}=\tau_{\eta_{i}}^{i}$ and $\eta_{i+1}=\inf \left\{k>\eta_{i}: x_{\tau_{k}^{i}}^{i} \in S_{0}\right\}$, with $\tau_{0}^{0}=0$, $\tau_{k}^{i}=\inf \left\{t>\tau_{k-1}^{i}: y_{t}^{i}=0\right\}$, for any $k \geq i \geq 1$.

The discrete time impulse control problem has been consider in Bensoussan [2], Stettner [37]. As seen later, it will be useful to consider an auxiliary problem in discrete time, for the Markov chain $X_{n}=x_{\tau_{n}}$, with the filtration $\mathbb{G}=\left\{\mathscr{G}_{n}, n \geq 0\right\}$, $\mathscr{G}_{n}=\mathscr{F}_{\tau_{n}}^{n-1}$. The impulses occurs at the stopping times $\eta_{k}$ with values in the set $\mathbb{N}=\{0,1,2, \ldots\}$ and are related to $\theta_{k}$ by $\eta_{i}=\inf \left\{k \geq 1: \theta_{k}=\tau_{k}\right\}$ for admissible controls $\left\{\theta_{k}\right\}$ and similarly for zero-admissible controls. Thus,

Definition 4.2. If $v=\left\{\left(\eta_{i}, \xi_{i}\right), i \geq 1\right\}$ is a sequence of $\mathbb{G}$-stopping times and $\mathscr{G}_{\eta_{i}{ }^{-}}$ measurable random variables $\xi_{i}$, with $\xi_{i} \in \Gamma\left(x_{\tau_{\eta_{i}}}\right), \eta_{i}$ increasing and $\eta_{i} \rightarrow+\infty$ a.s., then $v$ is referred to as an 'admissible discrete time' impulse control if $\eta_{1} \geq 1$. If $\eta_{i} \geq 0$ is allowed, this is referred as an 'zero-admissible discrete time' impulse control.

\subsection{Common Assumptions}

It is assumed that there are a running cost $f(x, y)$ and a cost-per-impulse $c(x, \xi)$ satisfying

$$
\begin{aligned}
& f: E \times \mathbb{R}^{+} \rightarrow \mathbb{R}^{+} \text {bounded and continuous, } \quad \alpha>0, \\
& c: E \times E \rightarrow\left[c_{0},+\infty\left[, c_{0}>0,\right. \text { bounded and continuous, }\right.
\end{aligned}
$$

where the discount factor is not used within the ergodic contest. Moreover, for any $x \in E$, the possible impulses must be in $\Gamma(x)=\{\xi \in E:(x, \xi) \in \Gamma\}$, where $\Gamma$ is a 
given analytic set in $E \times E$ such that for every $x$ in $E$ the following properties hold true

$$
\begin{aligned}
& \emptyset \neq \Gamma(x) \text { is compact }{ }^{2}, \quad \forall \xi \in \Gamma(x), \Gamma(\xi) \subset \Gamma(x), \quad \text { and } \\
& c(x, \xi)+c\left(\xi, \xi^{\prime}\right) \geq c\left(x, \xi^{\prime}\right), \quad \forall \xi \in \Gamma(x), \forall \xi^{\prime} \in \Gamma(\xi) \subset \Gamma(x) .
\end{aligned}
$$

Finally, defining the operator $M$

$$
M v(x)=\inf _{\xi \in \Gamma(x)}\{c(x, \xi)+v(\xi)\},
$$

it is assumed that

$M$ maps $C_{b}(E)$ into $C_{b}(E)$, and there exists a measurable

selector $\hat{\xi}(x)=\hat{\xi}(x, v)$ realizing the infimum in $M v(x), \forall x, v$.

Remark 4.1. (a) The last condition in (24) is to ensure that simultaneous impulses is never optimal. (b) (26) requires some regularity property of $\Gamma(x)$, e.g., see Davis [10]. (c) It is possible (but not necessary) that $x$ belongs to $\Gamma(x)$, actually, even $\Gamma(x)=E$ whenever $E$ is compact. However, an impulse occurs when the system moves from a state $x$ to another state $\xi \neq x$, i.e., it suffices to avoid (or not to allow) impulses that moves $x$ to itself, since they have a higher cost.

\section{Discounted Cost}

This section is an extension of [29] to a locally compact space $E$.

\subsection{HJB Equation}

The discounted cost of an impulse control (or policy) $\left.v=\left\{\left(\theta_{i}, \xi_{i}\right): i \geq 1\right)\right\}$ is given by

$$
J_{x, y}(v)=\mathbb{E}_{x, y}^{v}\left\{\int_{0}^{\infty} \mathrm{e}^{-\alpha t} f\left(x_{t}, y_{t}\right) \mathrm{d} t+\sum_{i=0}^{\infty} \mathrm{e}^{-\alpha \theta_{i}} c\left(x_{\theta_{i}}^{i-1}, \xi_{i}\right)\right\},
$$

where $\mathbb{E}_{x y}^{v}$ is the $P_{x y}^{v}$-expectation of the process under the impulse control $v$ with initial conditions $\left(x_{0}, y_{0}\right)=(x, y)$, and $x_{\theta_{i}}^{i-1}$ is the value of the process just before the impulse. Note that the process $\left\{y_{t}: t \geq 0\right\}$ is not subject to any impulse, and the condition $y_{\theta}=0$ determines admissibility of the impulse time $\theta$.

Thus, the optimal cost is defined by

\footnotetext{
2 compactness is not really necessary, but it is convenient
} 


$$
u(x, y)=\inf \left\{J_{x, y}(v): v \in \mathscr{V}\right\}, \quad \forall(x, y) \in E \times[0, \infty[
$$

and its associated auxiliary impulse control problem (referred to as the timehomogeneous' impulse control) with an optimal cost given by

$$
u_{0}(x, y)=\inf \left\{J_{x, y}(v): v \in \mathscr{V}_{0}\right\}, \quad \forall(x, y) \in E \times[0, \infty[.
$$

As with the optimal stopping time problems, since $u(x, y)=u_{0}(x, y)$ for any $x \in E$ and $y>0$, it may be convenient to write $u_{0}(x)=u_{0}(x, 0)$ as long as no confusion arrives.

The Dynamic Programming Principle shows (heuristically), see [29, Section 3] that

$$
u(x, y)=\mathbb{E}_{x y}\left\{\int_{0}^{\tau} \mathrm{e}^{-\alpha t} f\left(x_{t}, y_{t}\right) \mathrm{d} t+\mathrm{e}^{-\alpha \tau} \min \{M u, u\}\left(x_{\tau}, y_{\tau}\right)\right\},
$$

and

$$
\begin{aligned}
& u_{0}(x, y)=\mathbb{E}_{x y}\left\{\int_{0}^{\tau} \mathrm{e}^{-\alpha t} f\left(x_{t}, y_{t}\right) \mathrm{d} t+\mathrm{e}^{-\alpha \tau} u_{0}\left(x_{\tau}, y_{\tau}\right)\right\}, \quad y>0, \\
& u_{0}(x)=\min \left\{\mathbb{E}_{x 0}\left\{\int_{0}^{\tau} \mathrm{e}^{-\alpha t} f\left(x_{t}, y_{t}\right) \mathrm{d} t+\mathrm{e}^{-\alpha \tau} u_{0}\left(x_{\tau}\right)\right\}, M u_{0}(x)\right\}
\end{aligned}
$$

are the corresponding Hamilton-Jacobi-Bellman (HJB) equations, which are referred to as quasi-variational inequalities (QVI) in a weak form. Note that $M$ is an operator in the variable $x$ alone, so that $M u(x, y)=[M u(\cdot, y)](x)$. In any case, $\min \{M u, u\}\left(x_{\tau}, y_{\tau}\right)=\min \{M u, u\}\left(x_{\tau}, 0\right)$, because $y_{\tau}=0$. Also, both problems are related (logically) by the condition

$$
u(x, y)=\mathbb{E}_{x y}\left\{\int_{0}^{\tau} \mathrm{e}^{-\alpha t} f\left(x_{t}, y_{t}\right) \mathrm{d} t+\mathrm{e}^{-\alpha \tau} u_{0}\left(x_{\tau}\right)\right\}
$$

and so, if $u_{0}(x)$ is known then the last equality yields $u(x, y)$ and $u_{0}(x, y)$. The optimal cost $u_{0}(x)$ can be expressed as a discrete-time optimal impulse control similar to Bensoussan [2, Chapter 8, 89-132] (ignoring the constraint), but this not necessary for the analysis, since everything is based on the results obtained for the optimal stopping time problems discussed in section 3.

\subsection{Solving the QVI}

Define

$$
u^{0}(x, y)=\mathbb{E}_{x y}\left\{\int_{0}^{\infty} \mathrm{e}^{-\alpha t} f\left(x_{t}, y_{t}\right) \mathrm{d} t\right\}, \quad \forall(x, y) \in E \times \mathbb{R}^{+},
$$


This function $u^{0}$ is the cost of no intervention, i.e., when the controller choose not to apply any impulse to the system. Since all cost are supposed nonnegative, the interval

$$
C_{b}\left(u^{0}, Z\right)=\left\{v \in C_{b}\left(E \times \mathbb{R}^{+}\right): 0 \leq v \leq u^{0}\right\},
$$

for either $Z=E \times \mathbb{R}^{+}$or $Z=E$, contains the optimal cost either $u$ or $u_{0}$, given by either (28) or (29).

To find a solution to the QVIs (30) and (31) set $u^{0}=u_{0}^{0}=u^{0}$ and consider the schemes

$$
\begin{aligned}
& u^{n}(x, y)=\mathbb{E}_{x y}\left\{\int_{0}^{\tau} \mathrm{e}^{-\alpha t} f\left(x_{t}, y_{t}\right) \mathrm{d} t+\mathrm{e}^{-\alpha \tau} \min \left\{M u^{n-1}, u^{n}\right\}\left(x_{\tau}, 0\right)\right\}, \\
& u_{0}^{n}(x)=\min \left\{\mathbb{E}_{x 0}\left\{\int_{0}^{\tau} \mathrm{e}^{-\alpha t} f\left(x_{t}, y_{t}\right) \mathrm{d} t+\mathrm{e}^{-\alpha \tau} u_{0}^{n}\left(x_{\tau}\right)\right\}, M u_{0}^{n-1}(x)\right\},
\end{aligned}
$$

for $n \geq 1$, i.e., a sequence of optimal stopping times problems with constraint. Based on Theorem 3.1, each VI has a unique solution either $u(x, y)$ in $C_{b}\left(E \times \mathbb{R}^{+}\right)$or $u_{0}^{n}$ in $C_{b}(E)$ satisfying either/or

$$
\begin{aligned}
& u^{n}(x, y)=\inf _{\theta} \mathbb{E}_{x y}\left\{\int_{0}^{\theta} \mathrm{e}^{-\alpha t} f\left(x_{t}, y_{t}\right) \mathrm{d} t+\mathrm{e}^{-\alpha \theta} M u^{n-1}\left(x_{\theta}, 0\right)\right\}, \\
& u_{0}^{n}(x)=\inf _{\theta} \mathbb{E}_{x 0}\left\{\int_{0}^{\theta} \mathrm{e}^{-\alpha t} f\left(x_{t}, y_{t}\right) \mathrm{d} t+\mathrm{e}^{-\alpha \theta} M u_{0}^{n-1}\left(x_{\theta}\right)\right\},
\end{aligned}
$$

where the minimization is over all admissible (or zero-admissible) stopping times $\theta$.

As in [29, Thms 4.2 and 4.3], we have

Theorem 5.1. Let us suppose Assumption 2.1 and (23), (24), (26). Then each of the sequences of functions $\left\{u_{0}^{n}\right\}$ and $\left\{u^{n}\right\}$ defined above, is monotone decreasing to the unique solution $u$ in $C_{b}\left(u^{0}, E \times \mathbb{R}^{+}\right)$and the solution $u_{0}$ in $C_{b}\left(u^{0}, E\right)$, of the QVIs (30) and (31). Moreover, the estimate: there exist constants $C>0,0<r<1$ such that

$$
\left|u^{n}(x, y)-u(x, y)\right|+\left|u_{0}^{n}(x, y)-u_{0}(x, y)\right| \leq C r^{n}, \quad \forall(x, y) \in E \times \mathbb{R}^{+},
$$

for all $n \geq 1$, as well as the relations (32),

$$
\begin{aligned}
& u(x, y)=\inf _{\theta} \mathbb{E}_{x y}\left\{\int_{0}^{\theta} \mathrm{e}^{-\alpha t} f\left(x_{t}, y_{t}\right) \mathrm{d} t+\mathrm{e}^{-\alpha \theta} M u\left(x_{\theta}, 0\right)\right\}, \\
& u_{0}(x)=\inf _{\theta} \mathbb{E}_{x 0}\left\{\int_{0}^{\theta} \mathrm{e}^{-\alpha t} f\left(x_{t}, y_{t}\right) \mathrm{d} t+\mathrm{e}^{-\alpha \theta} M u_{0}\left(x_{\theta}\right)\right\},
\end{aligned}
$$

hold true, where the minimization is over (zero-)admissible stopping times $\theta$. Furthermore, $u^{n}$ and $u$ belong to the domain $\mathscr{D}\left(A_{x, y}\right) \subset C_{b}(E \times[0, \infty[)$ of the infinitesimal generator $A_{x, y}$, and $u(x, y)$ 


$$
\begin{gathered}
-A_{x, y} u(x, y)+\alpha u(x, y)+\lambda(x, y)[u(x, 0)-(M u(\cdot, 0))(x)]^{+}= \\
=f(x, y), \quad \forall(x, y) \in E \times \mathbb{R}^{+}, \\
-A_{x, y} u^{n}(x, y)+\alpha u(x, y)+\lambda(x, y)\left[u^{n}(x, 0)-\left(M u^{n-1}(\cdot, 0)\right)(x)\right]^{+}= \\
=f(x, y), \quad \forall(x, y) \in E \times \mathbb{R}^{+}, \forall n \geq 1,
\end{gathered}
$$

are equivalent to the corresponding QVI and VI.

Proof. Only a short idea of the main points in the proof are mentioned. First, a decreasing and concave mapping is defined with the expressions in (35), and following an argument similar to the one used in Hanouzet and Joly [16], the exponential convergence/estimate is proved and a fixed point (solving the QVIs) is obtained. At this point, the remaining assertions are obtained with a little more work.

In the following Theorem, all assertions are written for the optimal cost (28), but a similar result holds true for the other optimal cost (29), with the zero-admissible impulse controls.

Theorem 5.2. Under the assumptions as in Theorem 5.1, the unique solution of the QVI equation (30) is the optimal cost (28), i.e., $u(x, y)=\inf \left\{J_{x, y}(v): v \in \mathscr{V}\right\}$, for every $(x, y)$ in $E \times \mathbb{R}^{+}$. Moreover, the first admissible exit time of the continuation region provides an optimal impulse control.

Proof. The arguments are the same as in [29, Thms $4.4 \& 4.5]$, there are no changes in assuming only $E$ locally compact (instead of compact), only the compactness of $\Gamma(x)$ is necessary. Most of the discussion involves some martingale properties.

Note that if $u$ is the optimal cost then (1) the continuation region $[u<M u]$ is defined as all $(x, y)$ in $E \times \mathbb{R}^{+}$such that $u(x, y)<M u(x, 0)$, (2) the optimal jump-to is a Borel minimizer $\hat{\xi}(x)$ of $M u(x, 0)$, i.e., $x \mapsto \hat{\xi}(x)$ is a Borel functions from $E$ into $\Gamma(x)$ and $c(x, \hat{\xi}(x))+u(\hat{\xi}(x), 0)=M u(x, 0)$, for every $x$ in $E$., and (3) the first exit time of $[u<M u]$ is defined as

$$
\hat{\theta}(x, y, s)=\inf \left\{t>s: u\left(x_{t-s}, y_{t-s}\right)=M u\left(x_{t-s}, 0\right), \quad y_{t-s}=0\right\},
$$

and $\hat{\theta}(x, y, s)=\infty$ if $u\left(x_{t-s}, y_{t-s}\right)<M u\left(x_{t-s}, 0\right)$ for every $t>s$ such that $y_{t}=0$. Note that he Markov process $t \mapsto\left(x_{t-s}, y_{t-s}\right)$, for $t \geq s$, represents the initial condition $\left(x_{s}, y_{s}\right)=(x, y)$. Moreover, the continuity ensures that

$$
u\left(x_{\hat{\theta}(x, y, s)-s}, 0\right)=c\left(x_{\hat{\theta}(x, y, s)-s}, \hat{\xi}\left(x_{\hat{\theta}(x, y, s)-s}\right)\right)+u\left(\hat{\xi}_{\left.\left(x_{\hat{\theta}(x, y, s)-s}\right), 0\right)}\right.
$$

whenever $\hat{\theta}(x, y, s)<\infty$.

Therefore, the evolution under the above feedback (or Markov impulse control as in Definition 4.1-iv) and initial conditions $(x, y)$ is as follows:

(1) first $\theta_{1}=\hat{\theta}(x, y, 0)$ and $\xi_{1}=\hat{\xi}\left(x_{\theta_{1}}\right)$ when $\theta_{1}<\infty$ (we may use an isolated 'coffin' state $\partial$ to set $x_{\infty}=\partial$ and $\hat{\xi}(\partial)=\partial$ ),

(2) next $\theta_{k+1}=\hat{\theta}\left(\xi_{k}, 0, \vartheta_{k}\right)$, for any $k \geq 1$. 
This is an optimal admissible impulse control $\hat{v}=\left\{\left(\theta_{k}, \xi_{k}\right): k \geq 1\right\}$, which is proved in the same way as for the case $E$ compact.

\section{Ergodic Cost}

This section is an extension of [30] to a locally compact space $E$.

\subsection{Setting-up}

We define the average cost to be minimized, as

$$
\begin{aligned}
& J^{T}(0, x, y, v)=\mathbb{E}_{x y}^{v}\left\{\int_{0}^{T} f\left(x_{s}^{v}, y_{s}^{v}\right) \mathrm{d} s+\sum_{i} \mathbb{1}_{\theta_{i} \leq T} c\left(x_{\theta_{i}}^{i-1}, \xi_{i}\right)\right\}, \\
& J(x, y, v)=\liminf _{T \rightarrow \infty} \frac{1}{T} J^{T}(0, x, y, v),
\end{aligned}
$$

the ergodic control problem is to characterize

$$
\mu(x, y)=\inf _{v \in \mathscr{V}} J(x, y, v),
$$

and to find an optimal control. The auxiliary problem is concerned with

$$
\begin{aligned}
& \mu_{0}(x, y)=\inf _{v \in \mathscr{Y}_{0}} \tilde{J}(x, y, v), \quad \text { with } \\
& \tilde{J}(x, y, v)=\liminf _{n \rightarrow \infty} \frac{1}{\mathbb{E}_{x y}^{v}\left\{\tau_{n}\right\}} J^{\tau_{n}}(0, x, y, v),
\end{aligned}
$$

and $J^{\tau_{n}}(0, x, y, v)$ as in (36) with $T=\tau_{n}$. Actually, as seen later, $\mu(x, y)=\mu_{0}(x, y)$ is a constant.

The Dynamic Programming Principle shows (heuristically, see [30, Section 3] that, with $w_{0}(x)=w_{0}(x, 0)$,

$$
\begin{aligned}
& w_{0}(x)=\min \left\{\mathbb{E}_{x 0}\left\{\int_{0}^{\tau}\left[f\left(x_{t}, y_{t}\right)-\mu_{0}\right] \mathrm{d} t+w_{0}\left(x_{\tau}\right)\right\}, M w_{0}(x)\right\}, \\
& w_{0}(x, y)=\mathbb{E}_{x y}\left\{\int_{0}^{\tau}\left[f\left(x_{t}, y_{t}\right)-\mu_{0}\right] \mathrm{d} t+w_{0}\left(x_{\tau}\right)\right\},
\end{aligned}
$$

are the corresponding Hamilton-Jacobi-Bellman (HJB) equations in a weak form with two unknowns $\mu_{0}$ and $w_{0}$. Note that $M$ is an operator in the variable $x$ alone, so that $M w_{0}(x, y)=\left[M w_{0}(\cdot, y)\right](x)$ as given by (25). Also, both problems are related (logically) by the condition 


$$
w(x, y)=\mathbb{E}_{x y}\left\{\int_{0}^{\tau}\left[f\left(x_{t}, y_{t}\right)-\mu_{0}\right] \mathrm{d} t+w_{0}\left(x_{\tau}\right)\right\}
$$

and so, if $w_{0}(x)$ is known then the last/first equality yields $w(x, y)$ and $w_{0}(x, y)$. Recall that $\tau$ is defined by (7) and that since $w(x, y)=w_{0}(x, y)$ for any $x \in E$ and $y>0$, it may be convenient to write $w_{0}(x)=w_{0}(x, 0)$ as long as no confusion arrives. Note that the functions $w(x, y)$ and $w_{0}(x)$ may be called potentials, and a priori, they are not costs, but they are used to determine an optimal control.

\subsection{Solving the HJB}

An important point to mention is to remark that the HJB equation (39) is equivalent to

$$
w_{0}(x)=\min \left\{M w_{0}(x), \ell(x)-\mu_{0} \tau(x)+P w_{0}(x)\right\},
$$

where

$$
\ell(x)=\mathbb{E}_{x 0}\left\{\int_{0}^{\tau} f\left(x_{s}, y_{s}\right) \mathrm{d} s\right\}, \quad \tau(x)=\mathbb{E}_{x 0}\{\tau\}
$$

with $\tau$ as in (7), and in view of the property (4),

$$
P h(x)=\mathbb{E}_{x 0}\left\{h\left(x_{\tau}\right)\right\},
$$

defines the operator $P$ on $C_{b}(E)$. Note that (10) yields

$$
0 \leq \ell(x) \leq a_{2}\|f\|
$$

Moreover, from the Feller property of $x_{t}$ and the law of $\tau$, it follows that $\ell(x)$ is continuous.

In addition to the hypotheses of Sections 2 and 4, we assume that there exists a positive measure $m$ on $E$ such that

$$
m(E)>0 \quad \text { and } \quad P(x, U) \geq m(U), \quad \forall U \in \mathscr{B}(E),
$$

where $P(x, U)=\mathbb{E}_{x 0} \mathbb{1}_{U}\left(x_{\tau}\right)$, with $\tau$ defined by (7), and $\mathscr{B}(E)$ is the Borel $\sigma$-algebra on $E$.

Remark 6.1. From

$$
P(x, U)=\mathbb{E}_{x 0}\left\{\int_{0}^{\infty} \lambda\left(x_{t}, t\right) \exp \left(-\int_{0}^{t} \lambda\left(x_{s}, s\right) \mathrm{d} s\right) \mathbb{1}_{U}\left(x_{t}\right) \mathrm{d} t\right\} .
$$

and Remark 2.3, one can check that (45) is satisfied when the transition probability of $x_{t}$ has a density with respect to a probability on $E$ satisfying: for every $\varepsilon>0$ there exists $k(\varepsilon)$ such that 


$$
p\left(x, t, x^{\prime}\right) \geq k(\varepsilon)>0, \text { on } E \times[\varepsilon, \infty[\times E .
$$

This is the case, for instance, for periodic diffusion processes, see Bensoussan [1], and for reflected diffusion processes with jumps, see Garroni and Menaldi [14, 15] (which is also valid for reflected diffusion processes without jumps). Furthermore, a simple example for $E$ locally compact is provided by a pure jump process with generator

$$
A_{x} g(x)=b(x)\left\{\int_{E} g(z) q(x, \mathrm{~d} z)-g(x)\right\} .
$$

One can check that (45) is satisfied if, for instance, $0<k_{0} \leq \lambda(x, y) \leq k_{1}, 0<b_{1} \leq$ $b(x) \leq b_{2}, q(x, B) \geq m_{0}(B)$ for a positive measure $m_{0}$, with with $m_{0}(E)>0$.

Lemma 6.1. Under assumption (45), there exist a positive measure $\gamma$ on $E$, and a constant $0<\beta<1$ such that $P(x, B) \geq \tau(x) \gamma(B)$, for every $B \in \mathscr{B}(E)$, any $x \in E$, with $\tau(x) \gamma(E)>1-\beta$.

Theorem 6.1. Under Assumption 2.1 and (23), (24), (26), as well as (45), there exists a solution $\left(\mu_{0}, w_{0}\right)$ in $\mathbb{R}^{+} \times C_{b}(E)$ of (41), and therefore, of (39).

For details of the Lemma 6.1 and Theorem 6.1 proofs, note that Kurano [21, 22] results hold true for a locally compact space $E$, and refer to [30, Lem 4.1 and Thm 4.2]. For instance, the assumptions (45) and (11) imply

$$
P \mathbb{1}_{B}(x)=: P(x, B) \geq \tau(x) \gamma(x), \quad \forall B \in \mathscr{B}(E),
$$

with $\gamma(B)=m(B) / a_{2}$ and any $\beta$ in $] 0,1\left[\right.$ such that $1-\beta<m(E) a_{1} / a_{2}$. Now, the HJB equation (41) can be written as

$$
w_{0}(x)=\inf _{\xi \in \Gamma(x) \cup\{x\}}\left\{\ell(\xi)+\mathbb{1}_{\xi \neq x} c(x, \xi)-\mu_{0} \tau(\xi)+P w_{0}(\xi)\right\} .
$$

Since $P^{\prime}(x, \mathrm{~d} z):=P(x, \mathrm{~d} z)-\tau(x) \gamma(\mathrm{d} z)$ satisfies $P^{\prime}(x, E)<\beta<1$, the operator

$$
R v(x)=\inf _{\xi \in \Gamma(x) \cup\{x\}}\left\{\ell(\xi)+\mathbb{1}_{\xi \neq x} c(x, \xi)+P w_{0}(\xi)-\tau(\xi) \int_{E} v(z) \gamma(\mathrm{d} z)\right\}
$$

is a contraction on $C_{b}(E)$ having a unique fixed point $w_{0}$, and moreover, $w_{0} \geq 0$ because $\ell(x) \geq 0$ and $c(x, \xi)>0$. Thus, $\left(\mu_{0}, w_{0}\right)$ is a solution, where $\mu_{0}:=\gamma\left(w_{0}\right)$, the integral of $w_{0}$ with respect to $\gamma(\cdot)$ on $E$.

Remark 6.2. When $\lambda$ does not depends on $x$, the function $\tau(x)$ is constant and (41) is the HJB equation of a standard discrete time impulse control problem as studied in Stettner [37, Section 4] for $\Gamma(x)=\Gamma$ fixed.

Then, we have

Theorem 6.2. Under the assumptions as in Theorem 6.1, the constant $\mu_{0}$ obtained in Theorem 6.1 satisfies 


$$
\mu_{0}=\inf \left\{\tilde{J}(x, 0, v): v \in \mathscr{V}_{0}\right\}
$$

and there exists an optimal feedback control based on the exit times of the continuation region $\left[w_{0}<M w_{0}\right]$.

Proof. First, by means of Theorem 6.1 when $\Gamma(x)=\{x\}$ (which means "no control'), we show that there exists $(j, h) \in \mathbb{R}^{+} \times C_{b}(E)$ solution of

$$
h(x)=\ell(x)-j \tau(x)+P h .
$$

Note that assumption (45) implies that $P$ has a unique invariant probability denoted by $\zeta_{0}(\mathrm{~d} x)$, see the book Hernández-Lerma [17, Section 3.3, pp. 56-61].

Thus, there are two cases: $\mu_{0}=j$ and $\mu_{0}<j$. First, for $\mu_{0}=j$, from the equation for $h$ and the fact that $X_{n}=x_{\tau_{n}}$ is a Markov chain, we have

$$
\begin{aligned}
j=\liminf _{n} \frac{1}{\mathbb{E}_{x 0}\left\{\tau_{n}\right\}} \mathbb{E}_{x 0}\left\{\sum_{i=0}^{n-1} \ell\left(X_{i}\right)\right\}= \\
\quad=\liminf _{n} \frac{1}{\mathbb{E}_{x 0}\left\{\tau_{n}\right\}} \mathbb{E}_{x 0}\left\{\int_{0}^{\tau_{n}} f\left(x_{t}, y_{t}\right) \mathrm{d} t\right\}=\tilde{J}(x, 0, v),
\end{aligned}
$$

with $v=0$, i.e., no impulse at all. Then, as in [30, Thm 5.1] we have $\mu_{0} \leq \tilde{J}(x, 0, v)$, for every $v$ in $\mathscr{V}_{0}$, i.e., $\mu_{0} \leq j$. Therefore, if $\mu_{0}=j$ then

$$
\mu_{0}=\inf \left\{\tilde{J}(x, 0, v): v \in \mathscr{V}_{0}\right\}=j=\tilde{J}(x, 0,0),
$$

and $v=0$, i.e., 'no impulses at all', is optimal.

Next, the case $\mu_{0}<j$ is treated as in [30, Thm 5.1], with $\tilde{w}(x)=w_{0}(x)-h(x)$, $\tilde{\ell}(x)=\left(j-\mu_{0}\right) \tau(x), \tilde{w}=\min \{M \tilde{w}, \tilde{\ell}+P \tilde{w}\}$. Indeed, using the results in Bensoussan [2, Section 7.4, pp. 74-77], we show that this discrete time problem has an optimal control $\hat{v}=\left\{\left(\hat{\eta}_{i}, \hat{\xi}_{i}\right): i \geq 1\right\}$ given by

$$
\hat{\eta}_{i}=\inf \left\{n \geq \hat{\eta}_{i-1}: w_{0}\left(X_{n}\right)=M w_{0}\left(X_{n}\right)\right\},
$$

where $X_{n}$ is the controlled Markov chain and $\hat{\xi}_{i}=\hat{\xi}\left(X_{\hat{\eta}_{i}}\right)$ with a measurable selector $\hat{\xi}(x)$ realizing the infimum in $M w_{0}(x)$. This is translated in continuous time as $\hat{\theta}_{i}=$ $\tau_{\hat{\eta}_{i}}$ and $\hat{\xi}_{i}=\hat{\xi}\left(x_{\hat{\theta}_{i}}\right)$.

Remark 6.3. It is clear that the previous argument about $(j, h)$ shows that the hypothesis (5.1) in our previous paper [30] is not really necessary, and therefore, it is a small improvement on it.

Theorem 6.3. Under the assumptions as in Theorem 6.1, the constant $\mu_{0}$ obtained in Theorem 6.1 satisfies

$$
\mu_{0}=\inf \{J(x, 0, v): v \in \mathscr{V}\}=J(x, y, \hat{v}),
$$

where $\hat{v}$ is obtained by $\tau$-translations from the optimal control in Theorem 6.2. 
Proof. (sketch) The first step is to show that $w(x, y)$ defined by (40) satisfied

$$
-A_{x y} w(x, y)+\lambda(x, y)[w(x, 0)-M w(x, 0)]^{+}=f(x, y)-\mu_{0}
$$

Actually, this is not surprising in view of the results for the discounted case, but the proof is somewhat cumbersome, see [30, Proposition 5.5].

This implies that the process

$$
M_{T}=\int_{0}^{T}\left[f\left(x_{t}, y_{t}\right)-\mu_{0}\right] \mathrm{d} t+w\left(x_{T}, y_{T}\right), \quad T \geq 0
$$

is a submartingale, and the argument is completed as in [30].

Remark 6.4 (Ergodic cost: A more general ergodic assumption). The assumption (45) is not satisfied, in general, for diffusion processes in the whole space, and thus, it is perhaps, relatively restrictive. A 'localized' substitute for (45) could be the assumption:

(i) there exist a closed set $C$, an open set $D, C \subset D \subset E$, and a constant $\left.\beta_{0} \in\right] 0,1[$ as well as a probability $m$ satisfying $0<m(C)<1=m(D)$ and such that $P(x, B) \geq$ $\beta_{0} m(B)$, for every $B \in \mathscr{B}(E)$, any $x \in E$; and

(ii) there exist a continuous function $W: E \rightarrow[1, \infty[$, and constant $\beta \in] 0,1[$ such that $P W$ is continuous and $P W(x) \leq \beta W(x)+\beta_{0} \mathbb{1}_{C} \int_{C} W(z) m(\mathrm{~d} z)$, for every $x \in E$.

An adaptation of Jaskiewicz [19] allows us to obtain a solution $\left(\mu_{0}, w_{0}\right)$ of (41), with $w_{0}$ in the weighted-space

$$
C_{W}(E)=\left\{g \text { continuous and } \sup _{x}\left\{\frac{|g(x)|}{W(x)}\right\}<\infty\right\},
$$

and to obtain Theorem 6.2, under some additional technical assumptions. Also, Theorem 6.3 can be obtained under the additional assumption

$$
\mathbb{E}\left\{\mathrm{e}^{-k_{0} t} W\left(x_{t}\right)\right\} \leq W(x), \quad \forall x \in E, t>0,
$$

where $\lambda(x, y) \geq k_{0}>0$ for every $x, y$. A detailed analysis will be in a paper in preparation [31] together with examples satisfying the various assumptions. This analysis is based on several references (e.g., Hernández-Lerma and Lasserre [18], Meyn and Tweedie [33, 32], among others).

\section{Extension}

As in $[28,29,30]$ let us mention some possible extensions:

- A variable discount factor $\alpha(x, y)$ instead of $\alpha$ constant, as well as a finitehorizon cost. 
- Letting the discount factor $\alpha \rightarrow 0$ in the optimal discounted costs $u^{\alpha}(x, y)$ and $u^{\alpha}(x)=u^{\alpha}(x, 0)=u_{0}^{\alpha}(x, 0)$ we expect to obtain ergodic costs, e.g., if $\mu_{\alpha}=$ $\alpha u^{\alpha}(x)$ and $w_{0}^{\alpha}(x)=u^{\alpha}(x)-u^{\alpha}\left(x_{0}\right)$ then $\mu_{\alpha} \rightarrow \mu_{0}$ and $w_{0}^{\alpha}(x) \rightarrow w_{0}(x)$, but this is still something to be properly shown, when $\Gamma(x)$ is not reduced to a fixed compact.

- A quantify signal, e.g, $y_{t}$ has jumps back to $\{0,1,2\}$ instead of only $\{0\}$ with the following meaning: there are three classes of impulse controls $\mathscr{V}_{0} \subset \mathscr{V}_{1} \subset \mathscr{V} / 2$ that are enabled only and accordingly to the value of $y_{t}$ (some more details are necessary for a convenient example). In this case, instead (7), the signals are given by the functional

$$
\tau=\inf \left\{t>0: y_{t} \in I\right\},
$$

where a prototype is $I=\{0,1,2\}$. In this case, the Markov chain will include also $y_{\tau_{n}}$, i.e., $\left(Z_{n}, e_{n}\right)=\left(x_{\tau_{n}}, y_{\tau_{n}}, \mathrm{e}^{-\alpha \tau_{n}}\right)$. We may think that as the waiting-time passes (indicated or represented by the process $y_{t}$ ) the necessity of 'controlling' increases and impulses to other regions (that previously were not allowed) becomes enabled, i.e., when $i<y_{t}<i+1$ then only the class $\mathscr{V} i$ of impulse controls is available, which produces an impulse back to some $y=j<i+1$. Actually, a detailed example may be needed, and this is not discussed here.

In this case, jumps should be always backward, i.e., $y_{t}$ may jumps only to the values 0,1 or 2 that are smaller that the value of $y_{t}$. Certainly, what is accomplished for three values could be applied for any finite number of values, and perhaps 'extrapolate' to infinite many values (as long as they are isolated values). Thus, $\psi(x, y)$ makes sense for the optimal stopping time problem (without any changes!) but the analysis within the impulse control could give some interesting surprises.

- For stopping time problems, recall that several extensions are possible, in particular the use of data with polynomial growth (instead of bounded). However, there are some extra complications for the impulse control problems.

\section{Hybrid Models}

The state of a continuous-time hybrid model has a continuous-type variable $x$ (with cad-lag paths) and a discrete-type variable $n$ (with cad-lag piecewise constant paths). The 'signal' is represented by the 'jumps' of $n_{t}$, and in general, this signal enable any possible change in the setting of the model, not only the 'possibility of controlling' as studied in this paper (an others). The general idea is that the usual evolution of the system is described by the component $x_{t}$, and 'once in a while' (or under some specific conditions) a discrete transition (i.e., a jump of $n_{t}$ occurs) and everything may change, and the evolution continues thereafter. With this in mind, the signal (to act, e.g., to control the system as in our model) is given by the 'hitting time' of a set of states $S$, i.e., $\tau=\inf \left\{t>0:\left(x_{t}, n_{t}\right) \in S\right\}$, and this set $S$ plays the 
role of a 'set-interface', where the continuous-type and discrete-type variables exchange information. This set-interface may be given a priori or used as part of the parameters of control. In our previous 'control with constraint' presentation, the discrete-type component $n_{t}$ was ignored (because there are only on/off possibilities) and the continuous-type component $x_{t}$ is actually composed by two parts $\left(x_{t}, y_{t}\right)$, as they were called, the reduced state $x_{t}$ and the signal process $y_{t}$. Thus, in our model, the set-interface $S$ is $E \times\{0\}$, the same for every $n$ (which is ignored, as mentioned earlier).

To present the problem studied in this paper as a hybrid model the 'details' (a) and (b) of Assumption 2.1 are not mentioned, and instead, assumptions directly on the functional (7) and the signal (8) are imposed, e.g., at least it is assumed (9), but for ergodic cost, the condition (10) is required. Also, some continuity is needed, i.e.,

$$
(x, y) \mapsto \mathbb{E}_{x y}\left\{\mathrm{e}^{-\alpha \tau} \varphi\left(x_{\tau}\right)\right\} \text { and }(x, y) \mapsto \mathbb{E}_{x y}\left\{\int_{0}^{\tau} \mathrm{e}^{-\alpha t} f\left(x_{t}, y_{t}\right)\right\} \mathrm{d} t
$$

are continuous functions, for every $\varphi$ in $C_{b}(E)$ and $f$ in $C_{b}(E \times[0, \infty[)$. Most of the results in previous section are valid under these 'more general' assumptions, except those involving the specific form of infinitesimal generator $A_{y}$ (3). To be more specific, the following results can be extended under these more general hypotheses: Theorem 3.1, without (22), for optimal stopping; Theorem 5.1 (without the formula regarding the generator), and Theorem 5.2 for the discounted cost; Theorem 6.1 and Theorem 6.2 (but not Theorem 6.3) for the ergodic cost. For instance, if we assume (9) and that signals given by (8) then define the time-interval between jumps $T_{n}=$ $\tau_{n}-\tau_{n-1}$, which (conditionally to $x_{t}$ ) forms an independent, identically distributed sequence of random variables with a non-negative and bounded intensity $\Lambda(x, y)$. Hence, the initial 'signal process' (which is not necessarily equal to the time elapsed since the last signal) can be replaced to obtain an equivalent (in most aspects) model as the one in this paper.

Indeed, let us make an example of a similar situation, i.e., a signal process $\tilde{y}_{t}$ which is not equal to the process $y_{t}$, the 'time elapsed since the last signal'. In this example, the state is $(x, \tilde{y})$, the controller is allowed to 'control' (via an impulse) when $\tilde{y}=0$, however, $\tilde{y}_{t}$ has

$$
A_{z} \varphi(z)=\partial_{z} \varphi(z)+\Lambda(z)[q \varphi(0)+(1-q) \varphi(z / 2)-\varphi(z)],
$$

as its infinitesimal generator, with $0<q<1$, i.e., the process $z_{t}$ jumps at $s_{n}, \sigma_{n}=$ $s_{n+1}-s_{n}$ are IID having an intensity $\Lambda(z)$, and at the jump-times, $z_{s_{n}}=0$ with probability $q$ and $z_{s_{n}}=z_{s_{n}} / 2$ with probability $1-q$. In this case, the functional of interest is always the same (7), namely, $\tau=\inf \left\{t>0: \tilde{y}_{t}=0\right\}$, with the sequence of signals $\tau_{k+1}=\inf \left\{t>\tau_{k}: \tilde{y}_{t}=0\right\}, \tau_{0}=0$, which are not necessarily the sequence of jump-times $\left\{s_{n}\right\}$ of the process $\tilde{y}_{t}$. However, if

$$
F(t)=1-\exp \left(-\int_{0}^{t} \Lambda(s) \mathrm{d} s\right)
$$


is the law of $\sigma_{1}$ for $P_{0}$ then the convolution $F^{* n}(t)$ is the law of $s_{n}=\sum_{n} \sigma_{n}$ and the law of $\tau=\tau_{1}$ (when $\tilde{y}_{0}=0$ ) is given by

$$
P_{0}\{\tau \leq t\}=\sum_{n} F^{* n}(t) q(1-q)^{n-1}:=G(t),
$$

and the sequence of signals $\left\{\tau_{k}\right\}$ define another sequence $T_{k}=\tau_{k+1}-\tau_{k}$ of IID random variables with law $G(t)$. Hence, if we take $\lambda(t)=G^{\prime}(t) /(1-G(t))$ then the control problem for $\left(x_{t}, \tilde{y}_{t}\right)$ and $f(x)$ (i.e., independent of $\tilde{y}$ ) should be equivalent to the problem $\left(x_{t}, y_{t}\right)$, with $y_{t}$ constructed from $\lambda(y)$, since the discrete problems are identical for $\left(x_{t}, \tilde{y}_{t}\right)$ and $\left(x_{t}, y_{t}\right)$. It is clear that theses considerations can be extended to a similar model $\left(x_{t}, z_{t}\right)$

$$
A_{z} \varphi(z)=b(z) \partial_{z} \varphi(z)+\Lambda(z) \int_{\mathbb{R}^{+}}(\varphi(\zeta)-\varphi(z)) m(z, \mathrm{~d} \zeta),
$$

under suitable assumptions on the drift $b$ and the probability kernel $m(\mathrm{~d} \zeta, z)$.

Another kind of problem could have the constraint 'control is allowed at any jump of $z_{t}{ }^{\prime}$, with $x_{t}$ as the reduced state process and $z_{t}$ as the signal process. For this model, the condition, 'when the process $z_{t}$ jumps' is not exactly the same as 'when $z_{t}$ vanishes'. In other words, technically speaking, the full state of the system needs something else that the knowledge of $(x, z)$, i.e., we need to know $z_{t}$ and $z_{t-}$ to check if a jump has really occurred. Thus, if $z_{t}=\tilde{y}_{t}$ above, then we would have $\tau_{n}=s_{n}$, the jump-times of $z_{t}$. For the $\left(x_{t}, y_{t}\right)$ model (as well as for the hybrid model) presented in the above sections, the constraint "control is allowed only ..." 'when $y_{t}$ jumps' is exactly the same as saying 'when $y_{t}$ vanishes'. Nevertheless, we may have an infinitesimal generator like $A_{z}$ (of the piecewise deterministic process $z_{t}-$ or something else-) with a $b(z)>0$ and $m(\varphi, z)=\varphi(0)$, which is not exactly the process $y_{t}$ (the time elapsed since the last signal), but it has the property that $z_{t}=0$ iff $y_{t}=0$. Thus, for those type of processes, the constraint "control is allowed only when $z_{t}$ vanishes" is equivalent to "control is allowed only when $y_{t}$ vanishes".

Because of the particular meaning of our signal process $y_{t}$ as the "time elapsed since last signal', we obtain more detailed results than in the general hybrid model. Therefore, there are many generalization in various directions, e.g., in between to consecutive signals some other type of control could be allowed, signals of various types enabling particular types of controls may be given, and many other ways on how a continuous-type and a discrete-type variables may interact. Actually, much more details on the (hybrid) model are necessary to advance further in this discussion, and this is part of our book in preparation Jasso-Fuentes et al. [20], which follows some the problems discussed in Bensoussan and Menaldi [5, 6] and [27].

\section{References}

1. A. Bensoussan. Perturbation methods in optimal control. Gauthier-Villars, Montrouge, 1988.

2. A. Bensoussan. Dynamic programming and inventory control. IOS Press, Amsterdam, 2011. 
3. A. Bensoussan and J.L. Lions. Applications des inéquations variationnelles en contrôle stochastique. Dunod, Paris, 1978.

4. A. Bensoussan and J.L. Lions. Contrôle impulsionnel et inéquations quasi-variationnelles. Gauthier-Villars, Paris, 1982.

5. A. Bensoussan and J.L. Menaldi. Hybrid control and dynamic programming. Dynam. Contin. Discrete Impuls. Systems, 3(4):395-442, 1997.

6. A. Bensoussan and J.L. Menaldi. Stochastic hybrid control. J. Math. Anal. Appl., 249(1):261288, 2000. Special issue in honor of Richard Bellman.

7. P. Brémaud. Point Processes and Queues. Springer-Verlag, New York, 1981. Martingale dynamics.

8. P. Brémaud. Markov Chains. Springer-Verlag, New York, 1999. Gibbs fields, Monte Carlo simulation, and queues.

9. O.L.V. Costa, F. Dufour, and A.B. Piunovskiy. Constrained and unconstrained optimal discounted control of piecewise deterministic Markov processes. SIAM J. Control Optim., 54(3):1444-1474, 2016.

10. M.H.A. Davis. Markov models and optimization. Chapman \& Hall, London, 1993.

11. P. Dupuis and H. Wang. Optimal stopping with random intervention times. Adv. in Appl. Probab., 34(1):141-157, 2002.

12. D. Egloff and M. Leippold. The valuation of American options with stochastic stopping time constraints. Appl. Math. Finance, 16(3-4):287-305, 2009.

13. S.N. Ethier and T.G. Kurtz. Markov processes. John Wiley \& Sons Inc., New York, 1986. Characterization and convergence.

14. M.G. Garroni and J.L. Menaldi. Green functions for second order parabolic integrodifferential problems. Longman Scientific \& Technical, Harlow, 1992.

15. M.G. Garroni and J.L. Menaldi. Second order elliptic integro-differential problems. Chapman \& Hall/CRC, Boca Raton, FL, 2002.

16. B. Hanouzet and J.L. Joly. Convergence uniforme des itérés définissant la solution d'une inéquation quasi variationnelle abstraite. C. R. Acad. Sci. Paris Sér. A-B, 286(17):A735-A738, 1978.

17. O. Hernández-Lerma. Adaptive Markov control processes. Springer-Verlag, New York, 1989.

18. O. Hernández-Lerma and J.B. Lasserre. Further topics on discrete-time Markov control processes. Springer-Verlag, New York, 1999.

19. A. Jaskiewicz. A fixed point approach to solve the average cost optimality equation for semiMarkov decision processes with Feller transition probabilities. Comm. Statist. Theory Methods, 36(13-16):2559-2575, 2007.

20. H. Jasso-Fuentes, J.L. Menaldi, and M. Robin. Hybrid Control for Markov-Feller Processes. To appear, 2016.

21. M. Kurano. Semi-Markov decision processes and their applications in replacement models. Journal of the Operations Research, Society of Japan, 28(1):18-29, 1985.

22. M. Kurano. Semi-Markov decision processes with a reachable state-subset. Optimization, 20(3):305-315, 1989.

23. J. Lempa. Optimal stopping with information constraint. Appl. Math. Optim., 66(2):147-173, 2012.

24. J.P. Lepeltier and B. Marchal. Théorie générale du contrôle impulsionnel markovien. SIAM J. Control Optim., 22(4):645-665, 1984.

25. G. Liang. Stochastic control representations for penalized backward stochastic differential equations. SIAM J. Control Optim., 53(3):1440-1463, 2015.

26. G. Liang and W. Wei. Optimal switching at poisson random intervention times. Discrete and Continuous Dynamical Systems, Series B. To appear, see arXiv:1309.5608v2 [math.PR].

27. J.L. Menaldi. Stochastic hybrid optimal control models. In Stochastic models, II (Guanajuato, 2000), volume 16 of Aportaciones Mat. Investig., pages 205-250. Soc. Mat. Mexicana, México, 2001.

28. J.L. Menaldi and M. Robin. On some optimal stopping problems with constraint. SIAM J. Control Optim., 54(5):2650-2671, 2016. 
29. J.L. Menaldi and M. Robin. On some impulse control problems with constraint. SIAM J. Control Optim., 55(5):3204-3225, 2017.

30. J.L. Menaldi and M. Robin. On some ergodic impulse control problems with constraint. SIAM J. Control Optim., 56(4):2690-2711, 2018.

31. J.L. Menaldi and M. Robin. Remarks on ergodic impulse control problems with constraint. In preparation, 2019.

32. S.P. Meyn and R.L. Tweedie. Stability of Markovian processes iii. Advances in Applied Probability, 25(3):518-548, 1993. Foster-Lyapunov criteria for continuous time processes.

33. S.P. Meyn and R.L. Tweedie. Markov chains and stochastic stability. Cambridge University Press, London, 2nd edition, 2009.

34. J. Palczewski and L Stettner. Finite horizon optimal stopping of time-discontinuous functionals with applications to impulse control with delay. SIAM J. Control Optim., 48(8):4874-4909, 2010.

35. G. Peskir and A. Shiryaev. Optimal stopping and free-boundary problems. Birkhäuser Verlag, Basel, 2006.

36. M. Robin. Contrôle impulsionnel des processus de Markov. Thèse d'état, 1978, 353 pp. Link at https://hal.archives-ouvertes.fr/tel-00735779/document.

37. L. Stettner. Discrete time adaptive impulsive control theory. Stochastic Process. Appl., 23(2):177-197, 1986.

38. L. Stettner. On ergodic impulsive control problems. Stochastics, 18(1):49-72, 1986.

39. H. Wang. Some control problems with random intervention times. Advances in Applied Probability, 33(2):404-422, 2001. 